\title{
Platinum nanoparticles reduce ovariectomy-induced bone loss by decreasing osteoclastogenesis
}

\author{
Woon-Ki Kim ${ }^{1 *}$, Jin-Chun Kim*, \\ Hyun-Jung Park ${ }^{1}$, Ok-Joo Sul ${ }^{1}$, Mi-Hyun Lee ${ }^{1}$, \\ Ji-Soon $\mathrm{Kim}^{2}$ and Hye-Seon $\mathrm{Choi}^{1,3}$ \\ ${ }^{1}$ Department of Biological Sciences (BK21 Program) \\ and Immunomodulation Research Center \\ ${ }^{2}$ School of Material Science Engineering \\ University of Ulsan \\ Ulsan 680-749, Korea \\ ${ }^{3}$ Corresponding author: Tel, 82-52-259-1545; \\ Fax, 82-52-259-1694; E-mail, hschoi@mail.ulsan.ac.kr \\ *These authors contributed equally to this work. \\ http://dx.doi.org/10.3858/emm.2012.44.7.048
}

Accepted 24 April 2012

Available Online 24 April 2012

Abbreviations: BMM, bone marrow-derived macrophages; $\left[\mathrm{Ca}^{2+}\right]$, intracellular concentration of $\mathrm{Ca}^{2+}$; CTX-1, collagen-type I fragments; DCFH-DA, 2',7'-dichlorofluorescein diacetate; M-CSF, macrophagecolony stimulating factor; MNC, multinucleated cells; NAC, $\mathrm{N}$-acetylcysteine; NFAT2, nuclear factor of activated T-cells cytoplasmic 1; OC, osteoclast; OVX, ovariectomy; PtNP, platinum nanoparticles; RANKL, receptor activator of nuclear factor- $\mathrm{KB}$ ligand; ROS, reactive oxygen species; TRAP, tartrate resistant alkaline phosphatase

\begin{abstract}
Platinum nanoparticles (PtNP) exhibit remarkable antioxidant activity. There is growing evidence concerning a positive relationship between oxidative stress and bone loss, suggesting that PtNP could protect against bone loss by modulating oxidative stress. Intragastric administration of PtNP reduced ovariectomy (OVX)induced bone loss with a decreased level of activity and number of osteoclast (OC) in vivo. PtNP inhibited OC formation by impairing the receptor activator of nuclear factor- $\mathrm{KB}$ ligand (RANKL) signaling. This impairment was due to a decreased activation of nuclear factor- $\mathrm{K} B$ and a reduced level of nuclear factor in activated T-cells, cytoplasmic 1 (NFAT2). PtNP lowered RANKL-induced long lasting reactive oxygen species as well as intracellular concentrations of $\mathrm{Ca}^{2+}$
\end{abstract}

oscillation. Our data clearly highlight the potential of PtNP for the amelioration of bone loss after estrogen deficiency by attenuated $\mathrm{OC}$ formation.

Keywords: NFAT2; osteoclasts; osteoporosis; ovariectomy; platinum nanoparticles; RANK ligand

\section{Introduction}

Bone, a dynamic tissue, is remodeled by the balanced action of bone formation and bone resorption. Estrogen deficiency facilitates bone remodeling, resulting in enlargement of the remodeling space with increases in cortical porosity creating resorption areas on the trabecular surface. Excess bone resorption is primarily caused by increased osteoclastogenesis. The main action of estrogen is to reduce osteoclastogenesis (Weitzmann and Pacifici, 2006). Osteoclasts (OC) are responsible for bone resorption. OC are derived from hematopoietic cells of the monocyte/macrophage lineage, sharing some morphological and functional characteristics with macrophages. There are two essential molecules required for osteoclastogenesis generated by bone marrow mesenchymal cells: macrophage-colony stimulating factor (M-CSF) and receptor activator of nuclear factor- $\kappa B$ ligand (RANKL), a member of the tumor necrosis factor (TNF) family (Kong et al., 1999; Suda et al., 1999). The binding of RANKL to its receptor, RANK, induces and activates signaling pathways required for differentiation, including not only the generation of reactive oxygen species (ROS), but also the induction of nuclear factor in activated T-cells, cytoplasmic 1 (NFAT2) to initiate signals for OC differentiation (Cappellen et al., 2002). Activated transcription factors result in the expression of target genes, including tartrate resistant alkaline phosphatase (TRAP) and calcitonin receptor.

ROS participate in a signaling pathway of RANKL/ RANK interaction. RANKL stimulation generates ROS in OC cells (Lee et al., 2005), which express NADPH oxidase, a ROS-generating enzyme (Steinbeck et al., 1994). Since ROS play roles in OC differentiation and bone resorption, antioxidants are used therapeutically to protect against bone loss. Human studies have shown lowered levels of 
A

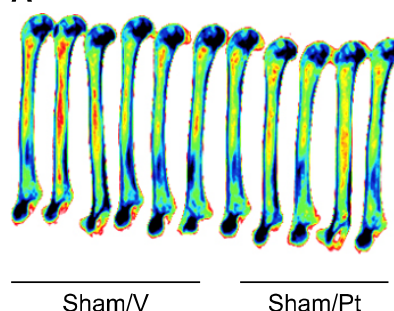

C

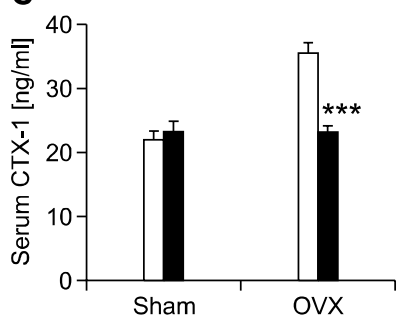

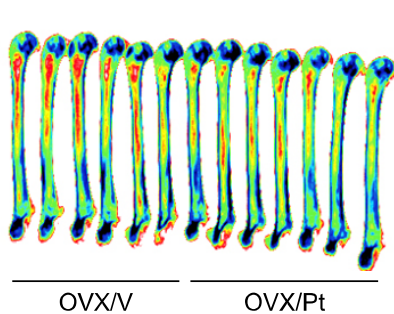

D

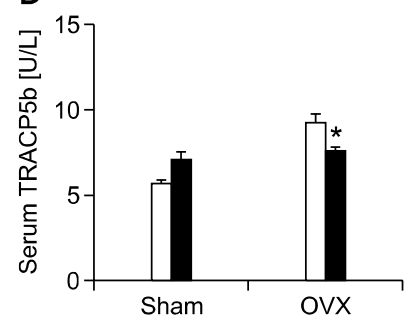

B

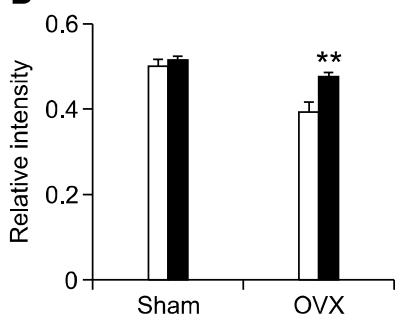

E

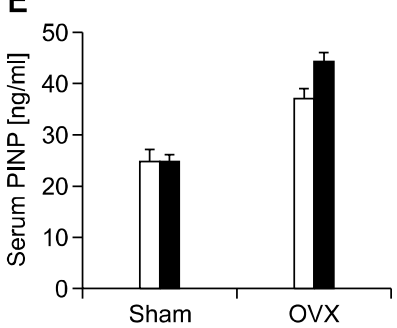

Figure 1. The effects of PtNP on OVX-induced bone loss in mice. The relative intensities of the distal metaphysic of the femur from $X$-ray radiograms (A) from vehicle (open bar) (OVX, $n=6$; sham, $n=6$ ), platinum nanoparticle-treated (closed bar) (OVX, $n=7$; sham, $n=5$ ) mice, 8 wk after OVX and sham surgery were measured using Image J program (B). Serum CTX-1 (C), TRACP5b (D). N-terminal propeptide of type I procollagen (PINP) (E) were measured by ELISA. Data were mean + SEM. ${ }^{*} P<0.05 ;{ }^{* *} P<0.01 ;{ }^{* * *} P<0.001$ compared with vehicle-treated mice. Differences between groups were analyzed by two-way ANOVA, followed by Bonferroni post tests to compare the effect of PtNP. There was no significant difference of serum PINP levels between vehicle- and PtNP-treated OVX.

antioxidants in osteoporotic patients (Basu et al., 2001; Maggio et al., 2003). The antioxidant $\mathrm{N}$-acetylcysteine (NAC) reduces bone loss in post-menopausal women (Sanders et al., 2007). In an animal model, ovariectomy (OVX) induces oxidative stress and results in bone loss. NAC and vitamin $C$ reduces OVX-induced bone loss in mice (Lean et al., 2005).

Platinum, a harmless metal, is widely used in a variety of industrial products, because of its catalytic activity. Since nanoparticles have unique chemical and biological properties, they are expected to have considerable potential in a wide range of biomedical applications. Their small sizes provide large surface areas on the nanometric scale, increasing their reactivity. Platinum nanoparticles (PtNP) eliminate anion radicals and hydrogen peroxide in a manner similar to superoxide dismutase and catalase (Kajita et al., 2007; Watanabe et al., 2009), suggesting a role as an antioxidant.

In the present study, we investigated whether PtNP have therapeutic effects in OVX-induced bone loss through their action on osteoclastogenesis.

\section{Results}

\section{PtNP protects against OVX-induced bone loss}

The femoral bone density of PtNP-administered mice was significantly higher than that of vehicle- treated mice after OVX, especially the distal metaphysis of the femur (Figures $1 \mathrm{~A}$ and $2 \mathrm{~B} ; P<$ 0.01 ). There were no significant differences between groups after sham surgery. Consistent with this finding, the PtNP-treated OVX mice had reduced levels of serum collagen-type I fragments (CTX-1), which is a bone resorption marker (Figure 1C; $P<$ 0.001). PtNP also decreased serum TRACP5b due to OVX (Figure 1D; $P<0.05$ ). However, the serum marker of bone formation, N-terminal propeptide of type I procollagen (PINP) was not significantly affected by PtNP treatment in OVX mice (Figure 1E). OVX also increased body weight compared to sham surgery (sham, $6.60 \pm 0.50 \mathrm{~g}$ vs. OVX, 12.02 $\pm 0.53 \mathrm{~g} ; P<0.001)$, and PtNP treatment reduced this elevation of body weight in OVX mice (7.82 \pm $0.45 \mathrm{~g} ; P<0.001$ ), showing that the elevation of bone mass by PtNP was not due to any increase of body mass.

\section{PtNP decreases OC formation}

To elucidate the mode of action of the PtNP on bone metabolism, we assessed its effect on BMM, which are OC precursors. PtNP inhibited OC formation from $B M M$ in a dose-dependent manner (Figures 2A and $2 \mathrm{~B}$ ), while total cell numbers were not significantly affected (data not shown), suggesting that the inhibitory effect was not due to cytotoxicity. Consistent with this result, transcripts of TRAP, calcitonin receptor, 

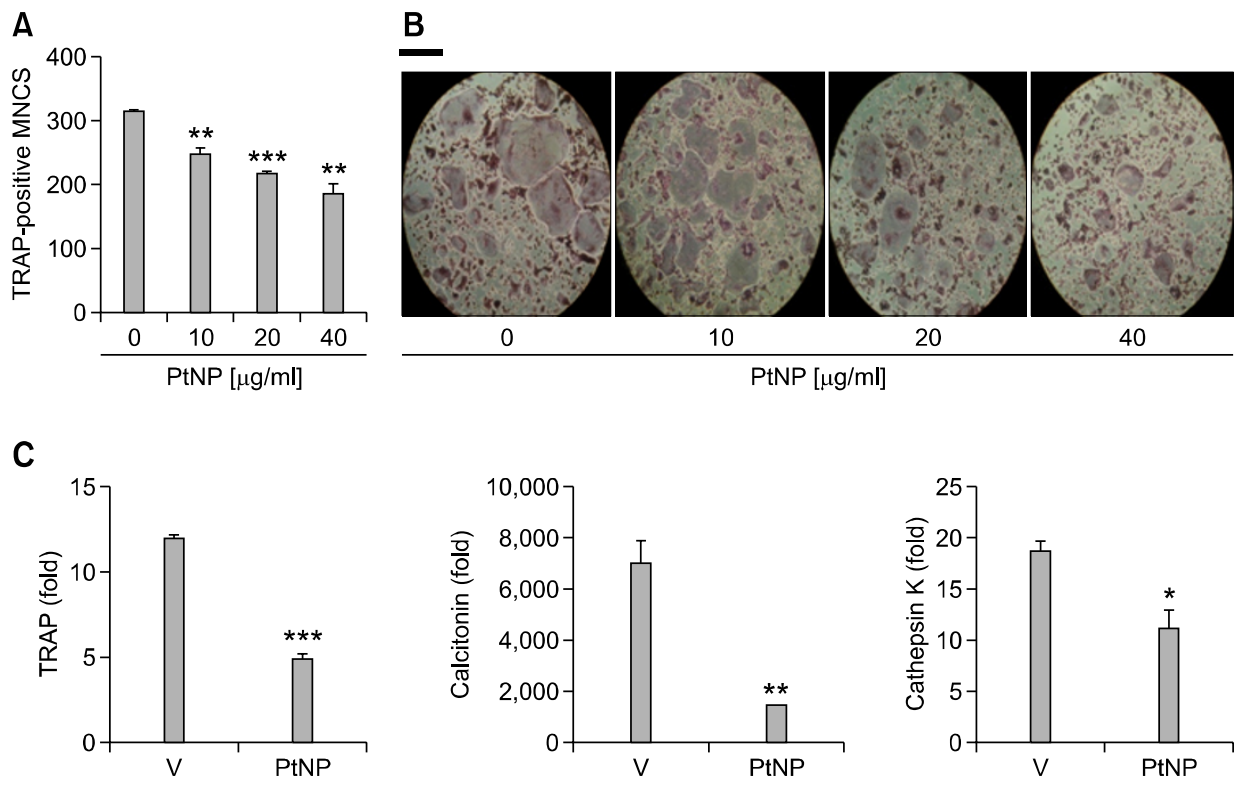

Figure 2. The effect of PtNP on RANKL-induced OC formation. BMMs $\left(8 \times 10^{3}\right.$ cells/well) were prepared and incubated with PtNP $(0,10,20,40 \mu \mathrm{g} / \mathrm{ml})$ in the presence of M-CSF $(20 \mathrm{ng} / \mathrm{ml})$ and RANKL $(40 \mathrm{ng} / \mathrm{ml})$. After 3 days, cells were fixed and stained for TRAP, and photographed. The number of TRAP-positive MNC per well was scored. ${ }^{* *} P<0.01$; ${ }^{* *} P<0.001$ compared with vehicle (A). Representative photos of A. Scale bar; $100 \mu \mathrm{m}$ (B). BMMs $\left(5 \times 10^{5}\right.$ cells/well) were incubated for $48 \mathrm{~h}$ with PtNP $(40 \mu \mathrm{g} / \mathrm{ml})$ in the presence of M-CSF and RANKL. Total RNA was isolated and subjected to qPCR analysis for TRAP, calcitonin receptor, and cathepsin K. The expression level before RANKL treatment was set at 1. ${ }^{*} P<0.05$; ${ }^{* *} P<0.01$; ${ }^{* * *} P$

$<0.001$ compared with vehicle $(\mathrm{C})$.

and cathepsin $\mathrm{K}$ were significantly reduced after RANKL stimulation of BMM in the presence of PtNP (Figure 2C). These results suggest that PtNP inhibit OC differentiation by acting in BMM.

During the differentiation of OCs, RANKL induces a key transcription factor, NFAT2, which plays critical and specific roles (Takayanagi et al., 2002). We examined whether PtNP affect the activation of these RANKL-induced signaling pathways. The exposure of BMM to RANKL resulted in induction of NFAT2. The expression level of NFAT2 in BMM after $48 \mathrm{hrs}$ of RANKL stimulation was greatly reduced in the presence of PtNP (Figure 3A). We also examined RANKL-induced NFAT2 expression by immunofluorescence staining using an anti-NFAT2 $\mathrm{Ab}$ (Figure 3B). In the absence of RANKL, almost no NFAT2 staining was detectible (first row), while in response to RANKL the expression of NFAT2 was found in the nucleus as well as in the cytoplasm. NFAT2 was superimposed in multinucleated cells characteristic for mature OC (second row). By contrast, treatment with PtNP resulted in not only a lowered number of $\mathrm{OC}$, but also an attenuated level of NFAT2 expression in comparison to vehicle treatment (second vs. third row).

Since ROS mediate RANKL signaling associated with NFAT2 activation in OC (Kim et al., 2010a), we tested whether PtNP affected RANKL-induced ROS generation. RANKL stimulated the formation of substantial levels of ROS in BMM, and again exogenous PtNP opposed this effect (Figure 3C). This finding implies that the reduction of osteoclastogenesis by PtNP could be due to decreased ROS production in response to RANKL.

Since ROS induces long lasting $\left[\mathrm{Ca}^{2+}\right]_{i}$ oscillations necessary for OC formation (Kim et al., 2010a), we also evaluated whether PtNP reduced RANKL-induced $\left[\mathrm{Ca}^{2+}\right]_{i}$ oscillations. Long term activation of BMM with RANKL for $48 \mathrm{hrs}$ induced the increase in the total $\left[\mathrm{Ca}^{2+}\right]$, whereas PtNP significantly attenuated it as shown in Figure 3D. Furthermore, the amplitude and frequency of $\left[\mathrm{Ca}^{2+}\right]_{\text {i }}$ oscillation response of each cell was also reduced by PtNP (Figure $3 \mathrm{E}$ ).

To investigate further inhibitory mechanism of PtNP in OC, we examined whether PtNP affected the RANKL-induced nuclear factor- $\kappa B$ (NF- $\kappa B$ ) activation. As shown in Figure $3 F$, crosslinking of RANKL to RANK stimulated NF- $\mathrm{KB}$ DNA binding activity (lane 5), and PtNP decreased this effect in a dose-dependent manner (lane 3,4). The specific binding was confirmed by competition assay using mutant competitor probes (lane 1).

Since RANKL binding to RANK activates three mitogen-activated protein kinases (MAPK) and Akt (Lee and Kim, 2003), we also examined whether PtNP affected the activation of these MAPKs and Akt. RANKL activated all 4 kinases in $5-15 \mathrm{~min}$ as shown in Figure 3G. PtNP attenuated activation of 

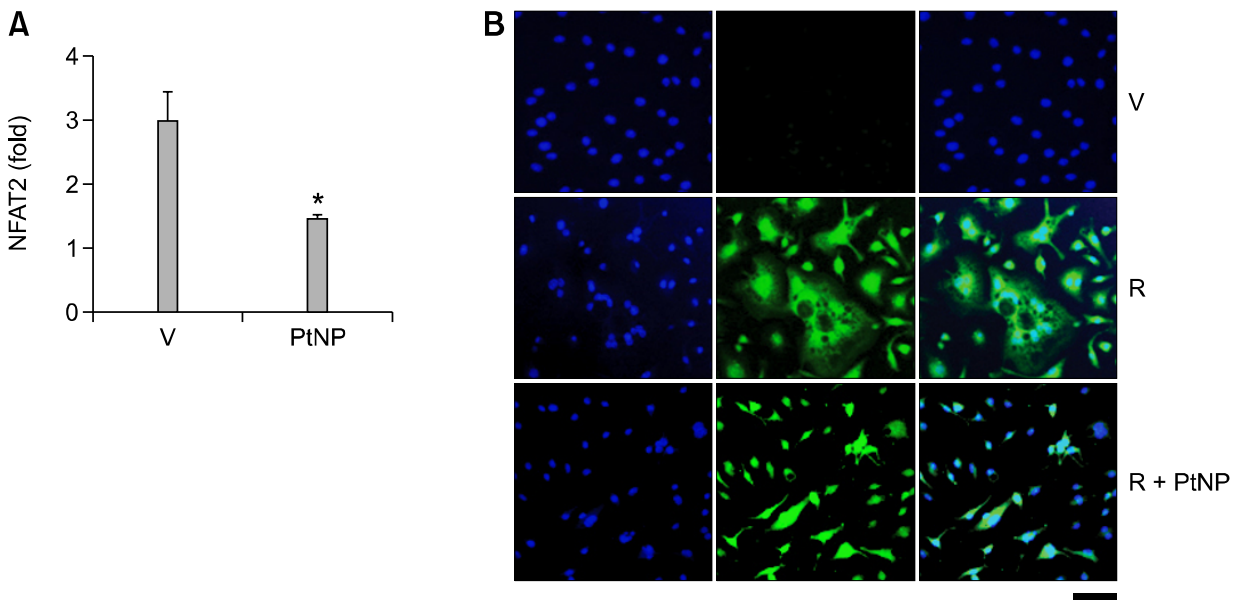

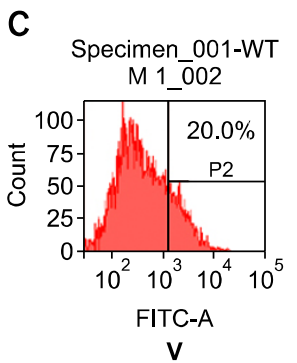

D

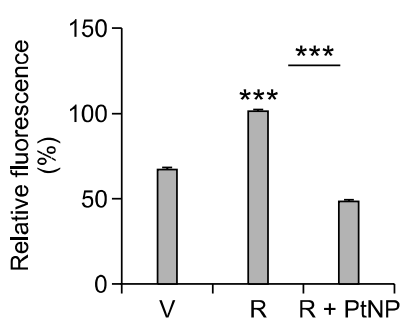

$\mathbf{F}$

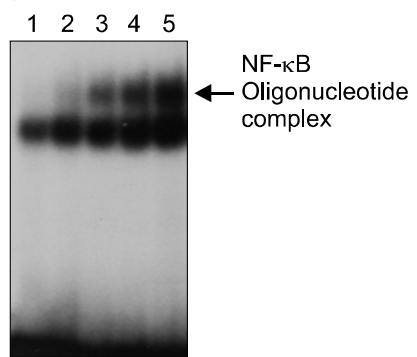

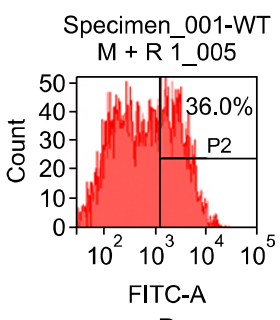

$\mathbf{R}$

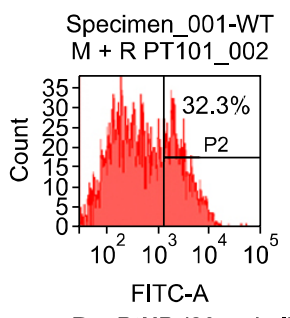

$R+\operatorname{PtNP}(20 \mu \mathrm{g} / \mathrm{ml})$
Specimen_001-WT $\mathrm{M}+\mathrm{R}$ PT2 201002

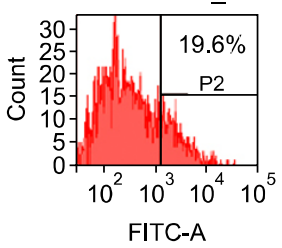

$R+\operatorname{PtNP}(40 \mu \mathrm{g} / \mathrm{ml})$
E

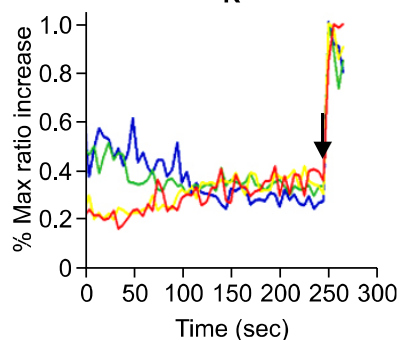

G

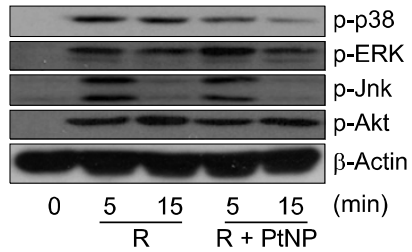

Figure 3. Impaired RANKL signaling by PtNP. BMM were stimulated with RANKL $(40 \mathrm{ng} / \mathrm{ml})$ and M-CSF $(20 \mathrm{ng} / \mathrm{ml})$ in the absence or presence of PtNP $(40 \mu \mathrm{g} / \mathrm{ml})$ for $48 \mathrm{hrs}$, and total RNA was extracted and subjected to $\mathrm{qPCR}$ analysis. The expression level before RANKL treatment was set at 1 . ${ }^{*} P<$ 0.05 compared with vehicle (A). BMM were incubated for 72 hrs in the presence of M-CSF only (first row) and M-CSF+RANKL (second row). When multinucleated cells with the morphologic features of OCs were observed, the cells were stained with Hoechest (first column) and anti-NFAT2 Ab (second column) to identify nuclear translocation. RANKL treatment induced multinucleated cells whose nucleus were superimposed with NFAT2 (second, third row) whereas OC precursors showed little staining of NFAT2 (first row). PtNP reduced the number of multinucleated cells with reduced level of NFAT2 staining (second vs. third row). Scale bar, $100 \mu \mathrm{m}$ (B). Intracellular levels of ROS upon stimulation with RANKL or RANKL + PtNP (40 $\mu \mathrm{g} / \mathrm{ml})$ in the presence of M-CSF for 48 hrs were determined in BMM cells using DCFH-DA. ROS levels were quantified by flow cytometry (C). Cells were loaded with fluo-4 AM for fluorescence (D). Cells were loaded with fluo-4 and Fura Red, and estimated as the ratio of fluorescence intensity of fluo-4 to Fura Red, and \% maximum ratio increase from the base line was plotted with an interval of $5 \mathrm{sec}$. Maximum ratio increase was obtained with the addition of $10 \mu \mathrm{M}$ ionomycin at the end of each experiment, indicated by an arrow. Each line indicated a different cell in the same field (E). BMM were stimulated with vehicle $V$ (lane 2), or RANKL (lane 5) in the absence or presence of PtNP $(20 \mu \mathrm{g} / \mathrm{ml}$, lane 4; $40 \mu \mathrm{g} / \mathrm{ml}$, lane 3) for $1 \mathrm{~h}$. A variant NF-KB (lane 1) was used as a negative control (F). Cytokine- and serum-starved BMM were exposed to RANKL (60 ng/ml). Phosphorylated forms of p38, ERK, JNK, and Akt were detected by Western blots (G). (A color figure can be viewed in the online version, which is available at www.e-emm.org). 
p38 at a modest degree, but not significant decrease in that of ERK, JNK, and Akt.

Taken together, our data indicate that PtNP caused defective RANKL signaling by decreased NFAT2 expression as well as impaired NF-kB activation.

\section{Discussion}

We demonstrated that exposure to PtNP reduced OVX-induced bone loss in vivo. PtNP opposed not only substantial reduction in femoral bone density, but also the elevation of CTX-1 and TRACP5b observed after OVX. Although OVX also increased body weight dramatically (Kim et al., 2010b), PtNP protected against this effect, so eliminating the possibility that the elevated bone mass is not due to increased body mass. The mechanism by which PtNP reduce the increase in body weight following OVX is now under investigation.

Bone is a dynamic tissue, involving a balance between bone formation by osteoblasts and resorption by OC (Goltzman, 2002). Postmenopausal osteoporosis may represent an imbalance in the favor of bone resorption over bone formation (Rodan, 1991). Higher bone density facilitated by PtNP after OVX appears to be due to inhibition of osteoclastogenesis. OCs are primarily responsible for bone resorption, and PtNP substantially inhibit OC formation. Gold nanoparticles suppressed the $N F-\kappa B$ activation and reduced the ROS level (Sul et al., 2010). Rutin acted as an antioxidant in OC to inhibit osteoclastogenesis via decreased the NF- $\mathrm{KB}$ activation (Kyung et al., 2008). Similar pattern was observed with PtNP-induced inhibitory effect in OC.

PtNP inhibit RANKL-induced OC formation in vitro by impairing RANKL signaling. PtNP did not display cytotoxicity to the BMM under these conditions. RANKL, a key differentiation factor for $O C$, is reported to induce the production of ROS (Kim et al., 2010a), indicating that ROS may take part in OC differentiation. We showed that PtNP attenuated ROS production and the total $\left[\mathrm{Ca}^{2+}\right]_{i}$ and $\left[\mathrm{Ca}^{2+}\right]_{i}$ oscillation stimulated by RANKL, and significantly decreased the expression of NFAT2. NFAT2 is an integral component of the RANKL signalling pathway during $O C$ differentiation. RANKL induces the expression and activation of NFAT2. Deficiencies of NFAT2 result in failure of OC differentiation, indicating that NFAT2 is a master switch controlling terminal differentiation of OCs (Takayanagi et al., 2002). A series of RANKL-induced signals leads to oscillation in the free $\left[\mathrm{Ca}^{2+}\right]_{\mathrm{i}}$, which triggers the later stage of $\mathrm{OC}$ differentiation by autoamplification of NFAT2 (Takayanagi et al., 2002). RANKL causes a long lasting increase in $\mathrm{ROS}$ and $\left[\mathrm{Ca}^{2+}\right]_{i}$ that is critical for OC differentiation (Kim et al., 2010a), suggesting that ROS may be responsible for the expression and the activation of NFAT2 on RANKL stimulation. Since PtNP decrease ROS, this decrease could be directly associated with decreased expression of NFAT2, consequently resulting in decreased NFAT2 activity. Blockade of NFAT activation by antioxidants has been demonstrated in several other cell types. NAC and catalase prevent vanadiuminduced NFAT activation mediated by $\mathrm{H}_{2} \mathrm{O}_{2}$ in fibroblasts (Huang et al., 2001), and adenovirally over-expressed Gpx-1 or manganese superoxide dismutase inhibits doxorubicin-induced nuclear NFAT translocation in rat cardiac cells (Kalivendi et al., 2005).

Our data indicate that PtNP act as antioxidants in $\mathrm{OC}$ and protect oxidative stress induced by OVX. This result is consistent with other findings that have demonstrated that the metal nanoparticle catalyzes some redox reactions. While we were preparing this, PtNP has been reported to decrease ROS via attenuating ROS-producing NOX family oxidases, NOX1 and NOX4, resulting in reduced osteoclastogenesis (Nomura et al., 2011). PtNP significantly inhibits heat-induced apoptosis in a dose-dependent manner. Superoxide has been suppressed to various extents (Yoshihisa et al., 2011). The PtNP complementarily stabilized with pectin decomposes hydrogen peroxide and consequently generate oxygen like catalase (Kajita et al., 2007). PtNP significantly extends the lifespan of wild-type nematodes and recovers the shortened lifespan of the mutant, which has excessive oxidative stress by acting as a superoxide dismutase/catalase mimetic (Kim et al., 2008). The potential use of PtNP as antioxidants calls for consideration of their possible biological toxicity. The cytotoxicity of PtNP has not been studied, but gold nanoparticles were nontoxic to mice and humans (Nam et al., 2003; Paciotti et al., 2004). Nanonization affects saturation solubility as well as surface area, increasing efficiency of delivery. It allows nanoparticles to reach high concentrations in tumors, and enhances the efficacy of X-ray therapy (Hainfeld et al., 2004). In therapeutic usage, the nonspecific uptake by macrophages could be a problem, due to the removal of more than $90 \%$ of an injected dose in 5 min (Muller, 1991). However, the transport of PtNP may not be a problem in OC whose precursor are macrophages. Our findings provide a rationale for the protective effect of PtNP on post menopause-associated bone loss. Further studies could lead to the use of PtNP in a novel therapy for osteoporosis. 


\section{Methods}

\section{Animals and study design}

Six-week-old C57BL/6J mice purchased from the Jackson Laboratory, and cared for at the University of Ulsan Immunomodulation Research Center (IRC) were subjected to OVX $(n=13)$ or sham operation $(n=11)$. PtNP were prepared every day as a solution of $200 \mu \mathrm{g} / \mathrm{ml}$ in phosphate-buffered saline (PBS). After surgery, vehicle $(n=12)$ or a solution of platinum nanoparticles $(5 \mu \mathrm{g} / \mathrm{g}$ of body weight/day) ( $n=12)$ was given intragastrically $(0.5 \mathrm{ml} / \mathrm{each})$ through an esophageal cannula to ensure delivery of the correct dose daily for 8 weeks. Bone density was analyzed. All mice were housed in the specific pathogen-free animal facility of the IRC and were handled in accordance with the guidelines of the Institutional Animal Care and Use Committee of the IRC. The standards were approved by that Committee (2010-021). Radiographic analysis of the femora was performed with a soft X-ray system (model CMB-2). The relative bone density of the distal metaphysis of the femur was measured by X-ray radiographic analysis with a real time image processing and measurement system (ZET-1). The accuracy and precision of these measurements were evaluated previously (Kyung et al., 2009). The femora of each animal were removed and preserved in $10 \%$ neutral buffered formalin. Bone remodeling marker was measured according to the manufacturer's directions (Immunodiagnostic Systems Inc.): serum collagen-type I fragments (CTX-1) by RatLaps EIA, serum TRACP 5 b by solid phase immunofixed enzyme activity assay, and N-terminal propeptide of type I procollagen (PINP) by a competitive EIA.

\section{OC formation}

Bone marrow cells were isolated from 4-5-week-old C57BL/6J mice as described (Lee et al., 2007). Femora and tibiae were removed aseptically and dissected free of adherent soft tissues. The bone ends were cut away, and the marrow cavity was flushed out from one end of the bone with $\alpha$-modified minimum essential medium ( $\alpha$-MEM) using a sterile 21-gauge needle. The bone marrow suspension was carefully agitated with a plastic Pasteur pipette to obtain single cells. These cells were washed twice and resuspended in $\alpha$-MEM containing $10 \%$ fetal bovine serum (FBS), and the suspension was incubated on plates along with M-CSF (20 ng/ml) (R \& D Systems, Inc., Minneapolis, $\mathrm{MN}$ ) for $16 \mathrm{~h}$. Non-adherent cells were then harvested, and cultured with M-CSF for two more days, at which time large populations of adherent monocyte/macrophage-like cells had formed on the bottom of the culture plates. The small number of non-adherent cells was removed by rinsing the dishes with PBS. The remaining adherent bone marrow-derived macrophages (BMM) were harvested and seeded in plates. Additional medium containing M-CSF and RANKL (40 ng/ml) (R \& D Systems, Inc.) was added, and the medium was replaced on day 3 . After incubation for the indicated times, the cells were fixed in $10 \%$ formalin for $10 \mathrm{~min}$, and stained for TRAP as described (Lee et al., 2007). The number of TRAP-positive multinucleated cells (MNC) (three or more nuclei) was scored.

\section{PtNP}

The PtNP were prepared by a novel single-step method (Park et al., 2009). The electrical explosion of platinum wire ( $3 \mathrm{kV}$ of charging voltage for $0.1 \mathrm{~mm}$ wire) in distilled water results in a highly dispersed suspension of PtNP with $-12.62 \mathrm{mV}$ of zeta potential. The average size of the platinum nanoparticles was found to be $(>100) \mathrm{nm}$ by using field emission scanning electron microscopy. The PtNP were sterilized under UV light for 1day, and adjusted to $5-20 \%(v / v) \alpha-M E M$ when treated.

\section{Quantitative PCR (qPCR)}

Total RNA isolated using TRIZOL reagent (Invitrogen, Carlsbad, CA) was reverse-transcribed with oligo-dT and Superscript I enzyme (Invitrogen), according to the manufacturer's instructions. Quantitative RT-PCR was carried out using SYBR Green 1 Taq polymerase (Qiagen, Hilden, Germany) and appropriate primers on a DNA Engine Opticon Continuous Fluorescence detection System (MJ Research Inc., Waltham, MA). The specificity of each primer pair was confirmed by melting curve analysis and agarose-gel electrophoresis. The housekeeping GAPDH gene was amplified in parallel with the genes of interest. The relative copy numbers compared to GAPDH were calculated using the expression $2^{-\Delta \Delta \mathrm{Ct}}$. The primer sequences used were as follows: 5'-ctgctcctagtgagcccaac-3' and 5'-ca gcaatcgacaaggagtga-3' (calcitonin receptor); 5'-gaccaccttg gcaatgtctctg-3' and 5'-tggctgaggaagtcatctgagttg-3' (TRAP);, 5'-tgaggcttctcttggtgtccatac-3' and 5'aaagggtgtcattactgcggg-3' (cathepsin K); 5'-aataacatgcgagccatcatc-3' and 5'-tcaccctg gtgttcttcctc-3' (NFAT2); 5'-acccagaagactgtggatgg-3' and 5'-cacattgggggtaggaacac-3' (GAPDH).

\section{Expression of NFAT2}

The BMM were incubated on culture plates with M-CSF with or without RANKL for $72 \mathrm{~h}$ to generate OCs. The cells were fixed in $10 \%$ neutral buffered formalin and permeabilized with $0.1 \%$ Triton $\mathrm{X}-100$. Immunofluorescence staining was performed using mouse anti-NFAT2 Ab (Santa Cruz Biotechnology) followed by FITC-conjugated anti-mouse IgG (eBioscience) and Hoechst (Sigma Chemical, St. Louis, MO). The subcellular localization of FITC-labeled NFAT2 was examined using a fluorescence microscope (Carl Zeiss, Germany).

\section{Intracellular reactive oxygen species (ROS) detection}

The intracellular formation of ROS was detected using the fluorescence probe, 2', 7'-dichlorofluorescein diacetate (DCFH-DA) (Molecular Probe). After the BMM were cultured under the different experimental conditions for $48 \mathrm{~h}$, the cells were harvested, trypsinized, suspended in PBS, loaded with DCFH-DA, and incubated at $37^{\circ} \mathrm{C}$ for $30 \mathrm{~min}$. The measurement of intracellular ROS was performed using a flow cytometry with a fluorescence-activated cell sorter (FACS) Calibur (Becton Dickinson). 


\section{Intracellular concentration of $\mathrm{Ca}^{2+}\left(\left[\mathrm{Ca}^{2+}\right]_{i}\right)$}

BMM were incubated with RANKL and M-CSF for $48 \mathrm{~h}$. For $\left[\mathrm{Ca}^{2+}\right]_{i}$ measurement, cells were incubated with $5 \mu \mathrm{M}$ fluo- 4 AM, $5 \mu$ M Fura Red AM, and $0.05 \%$ pluronic F127 for 30 min in serum-free DMEM as described (Takayanagi et al., 2002). Cells were incubated further with M-CSF for 20 min, and analyzed using a confocal microscope (Olympus). To estimate $\left[\mathrm{Ca}^{2+}\right]_{i}$ in single cells, the ratio of the fluorescence intensity of fluo-4 to fura red was calculated. The increase in the ratio from the basal level was divided by the maximum ratio increase obtained by adding $10 \mu \mathrm{M}$ ionomycin and was expressed as the \% maximum ratio increase. For measurement of total $\left[\mathrm{Ca}^{2+}\right]_{i}$ BMM cultured with RANKL and M-CSF for $48 \mathrm{~h}$ were loaded with fluo- 4 at $37^{\circ} \mathrm{C}$ for 30 min, further incubated at room temperature for additional $30 \mathrm{~min}$, and analyzed with an excitation/emission filter pair $(488 / 530 \mathrm{~nm})$

\section{EMSA}

BMM were stimulated with RANKL for $1 \mathrm{~h}$ and nuclear extracts were prepared. NF-KB-binding studies were performed using a double-stranded oligonucleotide (Santa Cruz Biotechnology, San Diego, CA) containing an NF-кB consensus binding site. The oligonucleotide or a variant was end-labeled with $\left[\gamma_{-}{ }^{32} \mathrm{P}\right]$ ATP using T4 polynucleotide kinase (Promega, Madison, WI). Five $\mu \mathrm{g}$ of each nuclear extract was incubated at $30^{\circ} \mathrm{C}$ for 20 min with $1 \mathrm{ng}$ of ${ }^{32} \mathrm{P}$-labeled probe in $10 \mu \mathrm{l}$ of binding buffer containing $1 \mu \mathrm{g}$ of poly (dl.dC), $15 \mathrm{mM}$ HEPES, $\mathrm{pH} 7.6,80 \mathrm{mM} \mathrm{NaCl}, 1$ mM EGTA, $1 \mathrm{mM}$ dithiothreitol, and $10 \%$ glycerol. DNAprotein complexes were visualized by electrophoresis on a native $5 \%$ polyacrylamide gel, vacuum-drying and autoradiography using an intensifying screen at $-80^{\circ} \mathrm{C}$.

\section{Western blotting}

Blots were probed with Abs against phosphorylated form of ERK, JNK, p38, and Akt (Cell Signaling) or $\beta$-actin (Santa Cruz Biotech.), and then incubated with the corresponding peroxidase-conjugated secondary Ab (Santa Cruz Biotech.).

\section{Statistical analysis}

All values are expressed as means \pm SEM. Student's $t$-test was used to evaluate differences between samples of interest and the corresponding controls. Differences between groups were assessed by two-way ANOVA followed by Bonferroni post tests. A probability $(P)$ value of less than 0.05 was considered statistically significant.

\section{Acknowledgements}

This work was supported by grants from the National Research Foundation funded by the Korean government (BRL-2009-0087350; KRF-2010-0002644).

\section{References}

Basu S, Michaelsson K, Olofsson H, Johnsson S, Melhus H. Association between oxidative stress and bone mineral density. Biochem Biophys Res Commun 2001;288:275-9

Cappellen D, Luong-Nguyen N, Bongiovanni S, Grenet O, Wanke C, Susa M. Transcriptional program of mouse osteoclast differentiation governed by the macrophage colony stimulating factor and the ligand for the receptor activator of NFKB. J Biol Chem 2002;277:21971-82

Goltzman D. Discoveries, drugs and skeletal disorders. Nat Rev Drug Discov 2002;1:784-96

Hainfeld JF, Slatkin DN, Smilowitz HM. The use of gold nanoparticles to enhance radiotherapy in mice. Phys Med Biol 2004;49:N309-15

Huang C, Ding M, Li J, Leonard SS, Rojanasakul Y, Castranova V, Vallyathan V, Ju G, Shi X. Vanadium-induced nuclear factor of activated $\mathrm{T}$ cells activation through hydrogen peroxide. J Biol Chem 2001;276:22397-403

Kajita M, Hikosaka K, litsuka M, Kanayama A, Toshima N, Miyamoto Y. Platinum nanoparticle is a useful scavenger of superoxide anion and hydrogen peroxide. Free Radic Res 2007;41:615-26

Kalivendi SV, Konorev EA, Cunningham S, Vanamala SK, Kaji EH, Joseph J, Kalyanaraman B. Doxorubicin activates nuclear factor of activated T-lymphocytes and Fas ligand transcription: role of mitochondrial reactive oxygen species and calcium. Biochem J 2005;389:527-39

Kim J, Takahashi M, Shimizu T, Shirasawa T, Kajita M, Kanayama A, Miyamoto Y. Effects of a potent antioxidant, platinum nanoparticle, on the lifespan of Caenorhabditis elegans. Mech Ageing Dev 2008;129:322-31

Kim MS, Yang Y, Son A, Tian YS, Lee S, Kang SW, Mualleum $S$, Shin DM. RANKL-mediated reactive oxygen species pathway that induces long lasting $\mathrm{Ca}^{2+}$ oscillations essential for osteoclastogenesis. J Biol Chem 2010a;285:6913-21

Kim YY, Kim SH, Oh S, Sul OK, Lee HY, Kim HJ, Kim SY, Choi HS. Increased fat due to estrogen deficiency induces bone loss by elevating monocyte chemoattractant protein (MCP-1) production. Mol Cells 2010b;9:277-82

Kong YY, Yoshida H, Sarosi I, Tan HL, Timms E, Capparelli C, Morony S, Olivereira-dos-Santos AJ, Van G, Itie A, Khoo W, Wakeham A, Dunstan CR, Lacey DL, Mak TW, Boyle WJ, Penninger JM. OPGL is a key regulator of osteoclastogenesis, lymphocyte development and lymph-node organogenesis. Nature 1999;397:315-23

Kyung TW, Lee JE, Shin HH, Choi HS. Rutin inhibits osteoclast formation by decreasing reactive oxygen species (ROS) and TNF- $\alpha$ by inhibiting activation of nuclear factor (NF)-кB. Exp Mol Med 2008;40:52-8

Kyung TW, Lee JE, Phan TV, Yu R, Choi HS. Osteoclastogenesis by bone marrow-derived macrophages is enhanced in obese mice. J Nutr 2009;139:502-6

Lean JM, Jagger CJ, Kirstein B, Fuller K, Chambers TJ. Hydrogen peroxide is essential for estrogen-deficiency bone loss and osteoclast formation. Endocrinology 2005;146:728-35 
Lee JE, Shin HH, Lee EA, Phan TV, Choi HS. Stimulation of osteoclastogenesis by enhanced level of MIP-1alpha in BALB/c mice. Exp Hematol 2007;35:1100-8

Lee NK, Choi YG, Baik JY, Han SY, Jeong D, Bae YS, Kim $\mathrm{N}$, Lee SY. A crucial role for reactive oxygen species in RANKL-induced osteoclast differentiation. Blood 2005;106: 852-9

Lee ZH, Kim HH. Signal transduction by receptor activator of nuclear factor kappa B in osteoclasts. Biochem Biophys Res Commun 2003;305:211-4

Maggio D, Barabani M, Pierandrei M, Polidori MC, Catani M, Mecocci P, Senin U, Pacifici R, Cherubini A. Marked decrease in plasma antioxidants in aged osteoporotic women: results of a cross-sectional study. J Clin Endocrinol Metab 2003;88:1523-7

Muller RH. Colloidal carriers for controlled drug delivery and targeting. Wissenschaftliche verlagsgesellschaft $\mathrm{mbH}$. 1991, CRC Press, Boston, MA

Nam JM, Thaxton CS, Mirkin CA. Nanoparticle-based bio-bar codes for the ultrasensitive detection of proteins. Science 2003;301:1884-6

Nomura M, Yoshimura Y, Kikuiri T, Hasegawa T, Taniguchi Y, Deyama Y, Koshira K, Sano H, Suzuki K, Inoue N. Platinum nanoparticles suppress osteoclastogenesis through scavenging of reactive oxygen species produced in RAW264.7 cells. J Pharmacol Sci 2011;117:243-52

Paciotti GF, Myer L, Weinreich D, Goia D, Pavel N, McLaughlin RE, Tamarkin L. Colloidal gold: a novel nanoparticle vector for tumor directed drug delivery. Drug Deliv 2004;11:169-83

Park EJ, Bae LH, Kim JS, Kwon YS, Kim JC, Choi HS, Chung $\mathrm{YH}$. Production and properties of Ag metallic nanoparticle fluid by electrical explosion of wire in liquid. J Korean Powder Metal Inst 2009;16:217-22

Rodan GA. Mechanical loading, estrogen deficiency, and the coupling of bone formation to bone resorption. J Bone Miner
Res 1991;6:527-30

Sanders KM, Kotowicz MA, Nicholson GC. Potential role of the antioxidant $\mathrm{N}$-acetylcysteine in slowing bone resorption in early post-menopausal women: a pilot study. Transl Res 2007;150:215

Steinbeck MJ, Appel WH, Verhoeven AJ, Karnovsky MJ. NADPH oxidase expression and in situ production of superoxide by osteoclast actively resorbing bone. J Cell Biol 1994;126:765-72

Suda T, Takahashi N, Udagawa N, Jimi E, Gillespie MT, Martin TJ. Modulation of osteoclast differentiation and function by the new members of the tumor necrosis factor receptor and ligand families. Endocrinol Rev 1999;20: 345-57

Sul OJ, Kim JC, Kyung TW, Kim HJ, Kim YY, Kim SH, Kim JS, Choi HS. Gold nanoparticle inhibited the receptor activator of nuclear factor- $\kappa B$ ligand (RANKL)-induced osteoclast formation by acting asan antioxidant. Biosci Biotechnol Biochem 2010;74:2209-13

Takayanagi H, Kim S, Koga T, Nishina H, Isshiki M, Yoshida $\mathrm{H}$, Saiura A, Isobe M, Yokochi T, Inoue J, Wagner EF, Mak TW, Kodama T, Taniguchi T. Induction and activation of the transcription factor NFATc1 (NFAT2) integrate RANKL signaling in terminal differentiation of osteoclasts. Dev Cell 2002;3:889-901

Watanabe A, Kajita M, Kim J, Kanayama A, Takahashi K, Mashino T, Miyamoto $Y$. in vitro free radical scavenging activity of platinum nanoparticles. Nanotechnology 2009;20: 455105(9pp)

Weitzmann MN, Pacifici R. Estrogen deficiency and bone loss: an inflammatory tale. J Clin Invest 2006;116:1186-94

Yoshihisa Y, Zhao QL, Hassan MA, Wei ZL, Furuichi M, Miyamoto Y, Kondo T, Shimizu T. SOD/catalase mimetic platinum particles inhibit heat-induced apoptosis in human lymphoma U937 and HH cells. Free Radic Res 2011;45: 326-35 